\title{
EDITORIAL
}

\section{ASPECTS RECENTS DES EXPLORATIONS ET DU TRAITEMENT DE L'IMPUISSANCE}

Ce numéro d'Andrologie réunit les principaux textes d'une réunion organisée à Paris le 15 Octobre 1991 au nom de la Société d'Andrologie de Langue Française. Seize exposés, en incluant ceux d'une table ronde consacrée à l'apport des injections intracaverneuses de prostaglandine El, avaient fait le point sur les aspects récents de l'exploration et du traitement de l'impuissance. J'y ai associé quelques textes supplémentaires se rapportant aux mêmes thèmes.

L'aspect le plus original de cette réunion, reflété dans les textes qui suivent, est d'avoir rassemblé sur l'estrade et dans la salle des représentants de toutes les disciplines impliquées dans l'impuissance : certes sexologues et urologues, mais aussi internistes, endocrinologues, diabétologues, neurophysiologistes, spécialistes du sommeil, psychiatres, pharmacologues et représentants de l'industrie pharmaceutique. Il en a résulté des confrontations et discussions souvent passionnantes, toujours enrichissantes et courtoises. A la satisfaction générale, au lieu des querelles surannées entre tenants d'un déterminisme essentiellement organique et tenants d'une origine essentiellement psychogène, un consensus s'établit presque immédiatement entre les différentes disciplines, reconnaissant aussi bien la fréquence des contributions organiques que la quasi-constance de l'intrication psychologique, la nécessité d'une réserve dans l'interprétation des résultats des explorations, presque toutes susceptibles d'être faussées par les effets du stress, et l'inadaptation d'une grande partie des thérapeutiques considérées classiques à l'attente des hommes impuissants et de leurs partenaires. Multidisciplinarité et respect mutuel ont permis aux participants à cette réunion de progresser vers un perception plus globale et plus juste de l'homme impuissant. Espérons que cette expérience ouvrira la voie à d'autres réunions de même genre.

Jacques BUVAT 